\title{
ELMy H-modes in JET helium-4 plasmas
}

\author{
D C McDonald ${ }^{1,12}$, J G Cordey ${ }^{1}$, E Righi' ${ }^{2,13}$, F Ryter $^{3}$, G Saibene ${ }^{2}$, \\ R Sartori $^{2}$, B Alper ${ }^{1}$, M Becoulet ${ }^{4}$, J Brzozowski ${ }^{5}$, I Coffey ${ }^{6}$, M de Baar $^{7}$, \\ P de Vries ${ }^{1,7}$, K Erents ${ }^{1}$, W Fundamenski ${ }^{1}$, C Giroud ${ }^{7}$, I Jenkins ${ }^{1}$, \\ A Loarte ${ }^{2}$, P J Lomas ${ }^{1}$, G P Maddison ${ }^{1}$, J Mailloux ${ }^{1}$, A Murari ${ }^{8}$,

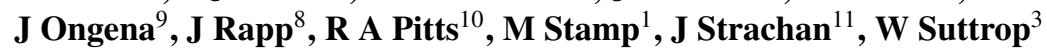 \\ and JET EFDA Contributors ${ }^{14}$ \\ ${ }^{1}$ Euratom/UKAEA fusion association, Culham Science Centre, Abingdon, Oxon, \\ OX14 3DB, UK \\ 2 EFDA Close Support Unit, D-85740 Garching, Germany \\ ${ }^{3}$ Max Planck Institut für Plasmaphysik Euratom Association, D-85740 Garching, Germany \\ ${ }^{4}$ Association Euratom-CEA, CEA Cadarache, F-13108, St Paul lez Durance, France \\ 5 Association Euratom-VR, KTH Royal Institute of Technology, Se-100 44 Stockholm, Sweden \\ ${ }^{6}$ Department of Physics, Queen's University, Belfast, UK \\ ${ }^{7}$ FOM-Instituut voor Plasmafysica 'Rijnhuizen', Associatie Euratom-FOM, Trilateral Euregio \\ Cluster, PO Box 1207, 3430 BE Nieuwegein, The Netherlands \\ ${ }^{8}$ EFDA-JET CSU, Culham Science Centre, Abingdon, Oxon, OX14 3EA, UK \\ ${ }^{9}$ LPP-ERM/KMS Association Euratom-Belgian State, Brussels, Belgium \\ ${ }^{10}$ CRPP Association Euratom-Confédération Suisse, École Polytechnique Fédérale de Lausanne, \\ CH-1015 Lausanne, Switzerland \\ ${ }^{11}$ PPPL, Princeton University, Princeton NJ, 08543, USA \\ E-mail: dmcd@jet.uk
}

Received 17 October 2003

Published 23 February 2004

Online at stacks.iop.org/PPCF/46/519 (DOI: 10.1088/0741-3335/46/3/007)

\begin{abstract}
ELMy H-modes in helium-4 plasmas provide valuable information on ELMy $\mathrm{H}$-mode physics as well as a possible early low activation operational phase for next-step tokamaks, such as ITER. With this in mind, a series of helium-4 H-mode experiments were performed on JET with pure helium-4 NBI auxiliary heating (up to $12 \mathrm{MW}$ ). A set of ELMy H-mode plasmas were produced, in both the Type I ELM regime and a second regime, which showed characteristics similar to the deuterium Type III regime, but with a reverse ELM frequency dependence on power. Sawteeth were also observed, and had similar behaviour to those seen in deuterium. Compared with deuterium plasmas, Type I ELMy $\mathrm{H}$-mode confinement is seen to be $28 \pm 6 \%$ poorer in helium- 4 plasmas and the $\mathrm{L}-\mathrm{H}$ power threshold $42 \pm 10 \%$ larger. This is the opposite of the behaviour predicted by experimental isotope mass scalings from hydrogenic plasmas.
\end{abstract}

\footnotetext{
12 Author to whom any correspondence should be addressed.

${ }^{13}$ Current address: European Commission, Brussels.

${ }^{14}$ See appendix of paper by Pamela J et al 2002 19th IAEA Fusion Energy Conf. (Lyon, October 2002). 
Comparison with a wider hydrogenic database, enables the effects of isotopic charge and mass to be studied independently.

\section{Introduction}

The study of H-modes in helium-4 plasmas offers valuable information for the understanding of edge and divertor physics, core confinement, pedestal physics, the L-H threshold, ELMs and sawteeth. Operation in helium-4 also provides a possible early low activation operational phase for next-step tokamaks, such as ITER. However, with the exception of a short campaign on the DIII-D facility [1], which used deuterium neutral beam injection (NBI), few helium $\mathrm{H}$-mode experiments have been carried out in large, diverted tokamaks. With this in mind, helium-4 experiments (with helium-4 plasmas up to $95 \%$ pure) were carried out on JET, with the Mark IIGB divertor. This paper presents results on the study of the L-H mode threshold, ELM and sawtooth behaviour, and energy confinement in helium-4 plasmas. They represent the first systematic study of the L-H threshold and ELMy H-modes in helium-4 plasmas in a large, diverted tokamak, and provide the first measures of the isotope charge dependence of the L-H threshold, ELMy H-mode confinement, ELM Type, and sawtooth period in ELMy $\mathrm{H}$-mode regimes. Results concerning the edge and divertor physics are reported elsewhere ([2], [3] and references therein).

This paper is structured as follows: section 2 outlines some relevant details of operating JET in helium-4 plasmas. Section 3 concerns the impact of helium plasmas on the conditions for L-H transitions. The behaviour of ELMs and sawteeth in helium-4 plasmas is discussed in section 4. The confinement properties of both Type I and Type III ELMy H-mode plasmas are studied in section 5. Finally, the results are summarized and discussed in section 6 . For the majority of the analyses, the behaviour of helium plasmas is compared with that of deuterium plasmas, which are used for the bulk of the operation in modern day tokamaks.

\section{Operation in helium}

Operation in helium presented many technical difficulties, most notably for NBI heating and divertor operation. For auxiliary heating, the NBI sources were converted to helium-4, and successfully coupled up to $12 \mathrm{MW}$ of power to the plasmas. However, the increased $\mathrm{L}-\mathrm{H}$ threshold in helium-4 (see section 3), limited the toroidal field and plasma current for which $\mathrm{H}$-modes could be achieved. Argon frosting of the Mark IIGB divertor enabled the pumping of helium but did reduce the number of discharges that could be run in a day. A small $(<2 \mathrm{MW})$ additional amount of minority hydrogen ICRH heating [4] was also used in some of the discharges studied in this paper.

Despite argon frosting, the rate at which the divertor pumped helium- 4 for the helium-4 discharges remained lower than the rate at which it pumped deuterium in the reference discharges [2]. This resulted in a steady increase in the divertor neutral density, except for plasmas with the lowest current and power. Although most of the deuterium in the near surface wall reservoir was removed by helium- 4 bombardment during the first helium- 4 discharge, significant amounts of deuterium remained more deeply embedded [5]. As a result, deuterium continued to be desorbed from the walls during high power Type I ELM phases, which is believed to be due to the large heat loads during ELM crashes at the strike points and main chamber wall surfaces. This resulted in a lower helium-4 ion purity in Type I ELMy H-modes (65-86\%), compared with that of the Type III ELMy H-modes (87-95\%). The lack 
of helium chemistry, resulting in the absence of deuterium chemical sputtering on the carbon surfaces of the first wall, meant that, in general, the helium plasmas had lower levels of carbon impurities $[2,6]$ than would be the case in hydrogenic plasmas.

Helium-4 concentrations were calculated from the $\mathrm{He}$ I and $\mathrm{D}_{\alpha}$ spectroscopic lines (essentially an edge measurement), with a constant concentration profile assumed. Photon efficiencies were also assumed to be constant. The low values of measured neutron emission (predominantly from the plasma core) in the helium- 4 discharges $\left(<1.1 \times 10^{11} \mathrm{~m}^{-3} \mathrm{~s}^{-1}\right.$ in all cases) were consistent with the helium- 4 concentration measurements from visible spectroscopy being within $20 \%$ of the true value. Thus, the relative errors in helium-4 concentration measurements for the discharges of this paper, have been estimated as having a standard deviation of $20 \%$. The errors in the spectroscopy, together with the consistency with neutron measurements, mean that this is the maximum uncertainty in the measurement.

Neutral beam power absorption, calculated by the PENCIL [7] code, has a reduced confidence due to the higher uncertainty in helium-4 cross-section measurements relative to hydrogenic ones [8]. Global thermal energy measurements, made by subtracting the fast particle energy calculated by PENCIL from the stored energy measured by magnetic coils, were similarly affected. These were found to be consistent with those calculated from density and temperature measurements. The standard deviations of the relative errors for the thermal energy measurements of the helium-4 discharges studied lie in the range of $8-11 \%$. Further analysis of plasma diagnosis from JET operation in helium-4 is reported elsewhere $[9,10]$.

\section{L-H threshold}

The $\mathrm{L}-\mathrm{H}$ threshold experiments were performed in JET, operating with the Mark IIGB divertor, in single null configurations with the ion-grad $B$ drift towards the X-point. Configuration, magnetic field, current and $q$-profiles were varied between discharges. The configurations were low shape $(\kappa=1.58-1.68, \delta=0.18-0.25)$, with the X-point typically $7-10 \mathrm{~cm}$ above the septum, but varying from $5 \mathrm{~cm}$ below to $13 \mathrm{~cm}$ above the septum for the dedicated X-point scans (section 3.3). Magnetic fields varied from 1.0-3.45 T, plasma currents from 1.0-3.2 T, and $q_{95}$ (the safety factor of the surface containing $95 \%$ of the poloidal flux of the last closed flux surface) from 2.9-3.8. Line averaged electron densities varied from $(1.5-3.9) \times 10^{19} \mathrm{~m}^{-3}$. The L-H power threshold was measured using a NBI only linear power ramp [11], with a ramp rate slow enough $\left(\mathrm{d} P_{\mathrm{NBI}} / \mathrm{d} t<1.5 \mathrm{MW} \mathrm{s}^{-1}\right.$ in all experiments, where $P_{\mathrm{NBI}}$ is the coupled NBI power) to ensure that, prior to the $\mathrm{L}-\mathrm{H}$ transition, the key plasma parameters were varying on a slow timescale compared with the plasma energy confinement time $\left(\mathrm{d} W_{\text {th }} / \mathrm{d} t \tau_{E}<0.2 W_{\text {th }}\right.$ and $\mathrm{d} \bar{n}_{\mathrm{e}} / \mathrm{d} t \tau_{E}<0.3 \bar{n}_{\mathrm{e}}$ in all cases, where $W_{\text {th }}$ is the plasma thermal energy, $\bar{n}_{\mathrm{e}}$ the line averaged electron density, and $\tau_{E}$ the energy confinement time). The relatively low divertor power loads of L-mode plasmas, together with the shortness of the auxiliary heating duration before the $\mathrm{L}-\mathrm{H}$ transition (typically $<2 \mathrm{~s}$ ), meant that deuterium concentrations were lower for the L-H threshold experiments than for the Type I ELMy H-mode confinement experiments (see section 5). For all the discharges, helium-4 concentrations were in the range 84-94\%.

\subsection{Density and power}

For the same $q_{95}(\approx 3.3)$ and configuration, helium- 4 and deuterium reference discharges were performed for a range of toroidal magnetic fields (1-3.2 T). During the power ramps for these shots, no gas was injected into the plasmas, but shots taken with and without argon frosting on the divertor tiles and the NBI cryopanels showed a natural variation in density. The small variations in plasma geometry in the experiments $\left(a=0.96, R_{0}=2.83-2.90\right.$; where $a$ is the 
plasma minor radius in $m$ and $R_{0}$ is the plasma major radius in $m$ ) mean that the dependence of the $\mathrm{L}-\mathrm{H}$ threshold on the major and minor radii cannot be studied. For deuterium plasmas, it has been shown [12] that the observed L-H power thresholds, derived from a multi-machine $\mathrm{H}$-mode power threshold database [13], are consistent with the expression

$$
P_{\mathrm{L}-\mathrm{H}}^{\mathrm{D} \text {, scal }}=0.87 \bar{n}_{\mathrm{e}}^{0.77} B_{0}^{0.92},
$$

where, $P_{\mathrm{L}-\mathrm{H}}$ is the loss power, $P_{\text {loss }}$, at the L-H transition in megawatts, $\bar{n}_{\mathrm{e}}$ the line averaged electron density in $10^{19} \mathrm{~m}^{-3}, B_{0}$ the vacuum magnetic field at the plasma centre in tesla, $P_{\text {loss }}=P_{\mathrm{IN}}-\dot{W}_{\text {th }}$ the loss power across the separatrix, and $P_{\mathrm{IN}}$ the total Ohmic and auxiliary power coupled to the plasma, and the mean values of minor and major radii for the $\mathrm{L}-\mathrm{H}$ threshold experiments at JET ( $a=0.96, R_{0}=2.87$ ) have been applied. Figure 1 shows the observed power thresholds plotted against equation (1). The deuterium reference discharges fit the scaling with a RMSE of $15.2 \%$. A direct fit of the helium-4 power threshold data to the line averaged density and magnetic field gives

$$
P_{\mathrm{L}-\mathrm{H}}^{\mathrm{He}-4}=(1.23 \pm 0.13) \bar{n}_{\mathrm{e}}^{0.77 \pm 0.17} B_{0}^{0.92 \pm 0.12} .
$$

Comparison with (1) shows that the power law scalings for density and field in helium-4 plasmas are identical with those of the scaling law for deuterium to two decimal places. With this fit, the helium- 4 data (nine discharges) has a RMSE of $11.6 \%$. Given the uncertainties in these measurements, the high degree of agreement in the exponents is clearly coincidental, but it is apparent that the power law scalings for density and field in helium-4 plasmas are consistent with those for deuterium plasmas within the error bars. However, the coefficient in (2) corresponds to a higher $\mathrm{L}-\mathrm{H}$ power threshold for a helium discharge compared to that predicted for a deuterium discharge by (1). We note that other, more recent, multi-machine

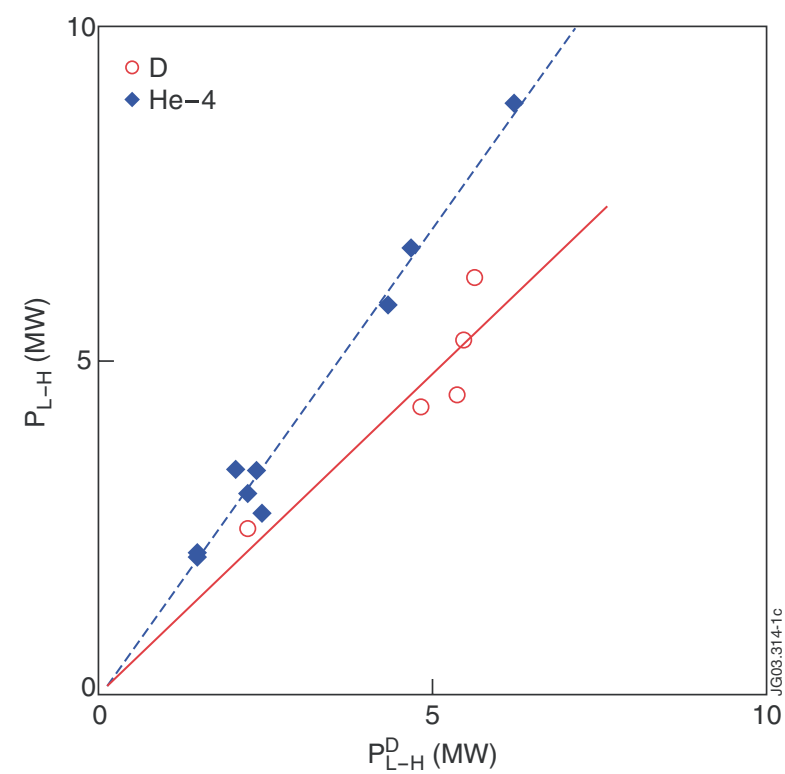

Figure 1. Comparison between the observed $\mathrm{L}-\mathrm{H}$ power threshold and the scaling law of equation (1) for NBI heated JET Mark IIGB helium-4 (solid blue diamonds) and deuterium reference (open red circles) plasmas. The observed $\mathrm{L}-\mathrm{H}$ power threshold, $P_{\mathrm{L}-\mathrm{H}}$, is on the vertical axis and the power threshold predicted for a pure deuterium plasma (1), $P_{\mathrm{L}-\mathrm{H}}^{\mathrm{H}}$, is on the horizontal axis. The solid red line represents the multi-machine scaling for deuterium plasmas (1). The broken blue line represents the fit to the helium data (2). 
scalings for the L-H threshold power of deuterium H-modes $[14,15]$ also fit the helium-4 and deuterium reference data of this paper, within error bars, but with a higher RMSE.

The increase of the L-H power threshold below a critical density, seen in earlier deuterium experiments on JET and several other tokamaks [16-21], was not observed in helium-4 plasmas. This indicates that if such a critical density exists for helium-4 discharges at JET, it lies below the densities studied $\left(\bar{n}_{\mathrm{e}}>1.5 \times 10^{19}\right.$ for all helium- $4 \mathrm{~L}-\mathrm{H}$ transitions). In deuterium JET plasmas, the critical density was found to be $\bar{n}_{\mathrm{e}}=(1-1.4) \times 10^{19}[21,22]$.

\subsection{Isotope effect}

As, when compared with hydrogenic plasmas, there is no evidence for the $\mathrm{L}-\mathrm{H}$ power threshold in helium-4 plasmas having a separate density and magnetic field scaling, the $\mathrm{L}-\mathrm{H}$ power threshold of the helium-4 plasmas studied may be taken to be of the form of equation (1). If this is the case, the $\mathrm{L}-\mathrm{H}$ power threshold of helium- 4 plasmas relative to deuterium plasmas may be written as

$$
P_{\mathrm{L}-\mathrm{H}}^{\mathrm{He}-4}=(1.42 \pm 0.10) P_{\mathrm{L}-\mathrm{H}}^{\mathrm{D}} \cdot
$$

The $\mathrm{L}-\mathrm{H}$ threshold is seen to be around $42 \%$ higher than that of deuterium. A log-linear fit with atomic charge and mass, including the earlier hydrogen, deuterium, deuterium-tritium and tritium data $[23,24]$, results in

$$
P_{\mathrm{L}-\mathrm{H}}=M^{-1.1} Z^{1.6} P_{\mathrm{L}-\mathrm{H}}^{\mathrm{D}, \text { scal }}
$$

where, $M$ and $Z$ are the mean isotope mass and charge, scaled to that of a proton. Thus, the power threshold for a helium-4 plasma would be significantly (52\%) below that of its hydrogen equivalent. Hence, for the low activation phase of a next step machine, the experiments suggest that the H-mode would be easier to access in helium-4 plasmas than in hydrogen ones.

Although equation (4) provides a convenient empirical formula for describing the $\mathrm{L}-\mathrm{H}$ power threshold in hydrogenic and helium plasmas, it is not clear that an isotope charge power law is generally appropriate. If $\mathrm{L}-\mathrm{H}$ transitions are dominated by the physics of fully ionized plasmas, such a formulation would seem to be justified. However, if $\mathrm{L}-\mathrm{H}$ transitions are dominated by atomic physics, a different isotope dependence may be expected.

Kiviniemi et al [25] have shown qualitative agreement between the observed mass and charge isotope dependence of the $\mathrm{L}-\mathrm{H}$ power threshold and a model where a neoclassically driven edge electric field stabilizes edge turbulence through $E \times B$ shear. Similarly, Hidalgo et al [26] have shown qualitative agreement between the observed increase in the $\mathrm{L}-\mathrm{H}$ power threshold of helium-4 plasmas relative to deuterium ones and a model based on turbulent transport, which is self-regulated by fluctuations.

\subsection{Plasma configuration}

Following JET Mark IIGB deuterium plasma experiments [27, 28], where the height of the $\mathrm{X}$-point above the septum was shown to alter the L-H power threshold by a factor of 2, a similar scan was repeated in helium-4. Figure 2 shows the range of configurations studied, with X-point heights varying between $5 \mathrm{~cm}$ below and $13 \mathrm{~cm}$ above the septum. The trend seen in deuterium was reproduced in helium-4 plasmas (figure 3), with the $\mathrm{L}-\mathrm{H}$ power threshold with the X-point on the septum being close to half that of $\mathrm{L}-\mathrm{H}$ transitions with the $\mathrm{X}$-point $6 \mathrm{~cm}$ above the septum. $P_{\mathrm{L}-\mathrm{H}}(0 \mathrm{~cm}) / P_{\mathrm{L}-\mathrm{H}}(6 \mathrm{~cm})=0.54$ in helium- 4 and $P_{\mathrm{L}-\mathrm{H}}(0 \mathrm{~cm}) / P_{\mathrm{L}-\mathrm{H}}(6 \mathrm{~cm})=0.61$ in the deuterium references for the dedicated discharges. For X-points more than $6 \mathrm{~cm}$ above the septum, the dependence disappears. This behaviour is not understood but, as earlier JET results in deuterium [27] showed an associated fall in the edge electron temperature immediately prior 


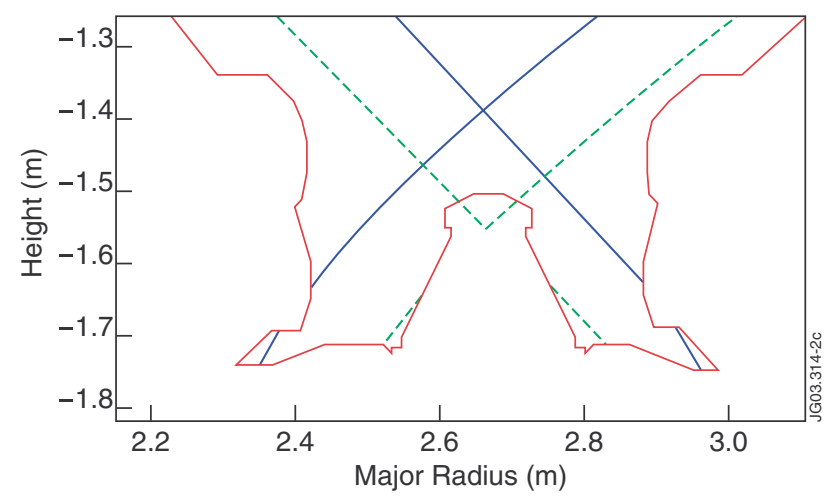

Figure 2. JET Mark IIGB divertor region for the range of configurations used in the L-H power threshold X-point scan. The height of the X-point varies from $5 \mathrm{~cm}$ below the septum (broken green line) to $13 \mathrm{~cm}$ above it (solid blue line).

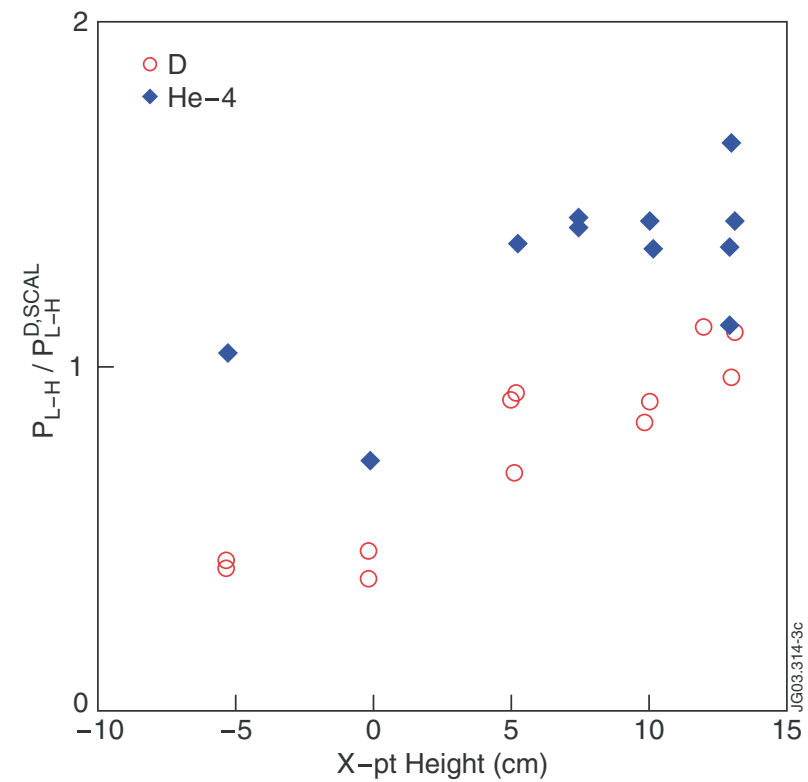

Figure 3. Impact of the $\mathrm{X}$-point height on the $\mathrm{L}-\mathrm{H}$ power threshold for NBI heated helium- 4 (solid blue diamonds) and deuterium reference (open red circles) JET Mark IIGB divertor plasmas.

to the $\mathrm{L}-\mathrm{H}$ transition for the same edge density and field, it is inconsistent with transition models based solely on fully ionized plasma physics. As such, the results would appear to support models involving neutral penetration, although such models must explain why helium-4 and deuterium plasmas show similar trends in threshold dependence when they possess different ionization properties. Related to this point, the plasma edge measurements for the helium-4 L-mode plasmas [2] indicated that the electron density and temperature profiles in the scrapeoff-layer were similar to their deuterium references. As no atomic physics is included in either the Kiviniemi model [25] or the work of Hidalgo [26], neither can explain the observed dependence on X-point height. However, the former finds that the predominant mechanism for the generation of the radial electric field is through ion orbit loss, which could clearly be 
dependent on neutrals. At present, though, this model simply replaces ions striking the walls and limiter with a source at the separatrix.

\section{ELM and sawtooth behaviour}

\subsection{Type I ELMs}

Type I ELMs were obtained in a range of configurations and currents $\left(1.0 \leqslant I_{\mathrm{p}} \leqslant 2.0\right.$; where $I_{\mathrm{p}}$ is the plasma current in mega-ampere). The ELM frequencies were relatively low $(15-45 \mathrm{~Hz})$. In all cases, the ELM crashes were clearly defined peaks with strong correlation between the inner and outer divertor $\mathrm{He}_{\mathrm{I}}$ line emission. Figure 4 shows the results of a NBI only power scan for a $1 \mathrm{MA} / 1 \mathrm{~T}$ helium- 4 plasma in a low triangularity $(\delta \approx 0.25)$, standard septum clearance (X-point $7 \mathrm{~cm}$ above the septum) configuration. As the injected NBI power increases across the scan from 6.3 to $10.1 \mathrm{MW}$, the ELM frequency increases monotonically from 17 to $50 \mathrm{~Hz}$. Thus, the ELM behaviour is in accordance with that shown in Type I ELMs in hydrogenic gases $[29,30]$. During these Type I ELM crashes in helium-4, the energy loss per ELM, $\Delta W_{\mathrm{ELM}}$ lay in the range $\Delta W_{\mathrm{ELM}} / W_{\mathrm{th}}=5-9 \%$, and the time averaged power loss per ELM, $P_{\mathrm{ELM}}$, in the range $P_{\mathrm{ELM}} / P_{\text {loss }}=16-25 \%$. For the deuterium reference Type I ELMy H-modes, the energy loss per ELM lay in the range $\Delta W_{\mathrm{ELM}} / W_{\text {th }}=4-9 \%$, and the time averaged power loss per ELM in the range $P_{\mathrm{ELM}} / P_{\text {loss }}=20-33 \%$. Although the values of $\Delta W_{\mathrm{ELM}} / W_{\mathrm{th}}$ for the helium-4 discharges lie within the scatter of those observed in earlier deuterium experiments on JET [31], the limited range of helium-4 discharges with pedestal data mean that it has not been possible to assess the fit of the data to specific models.

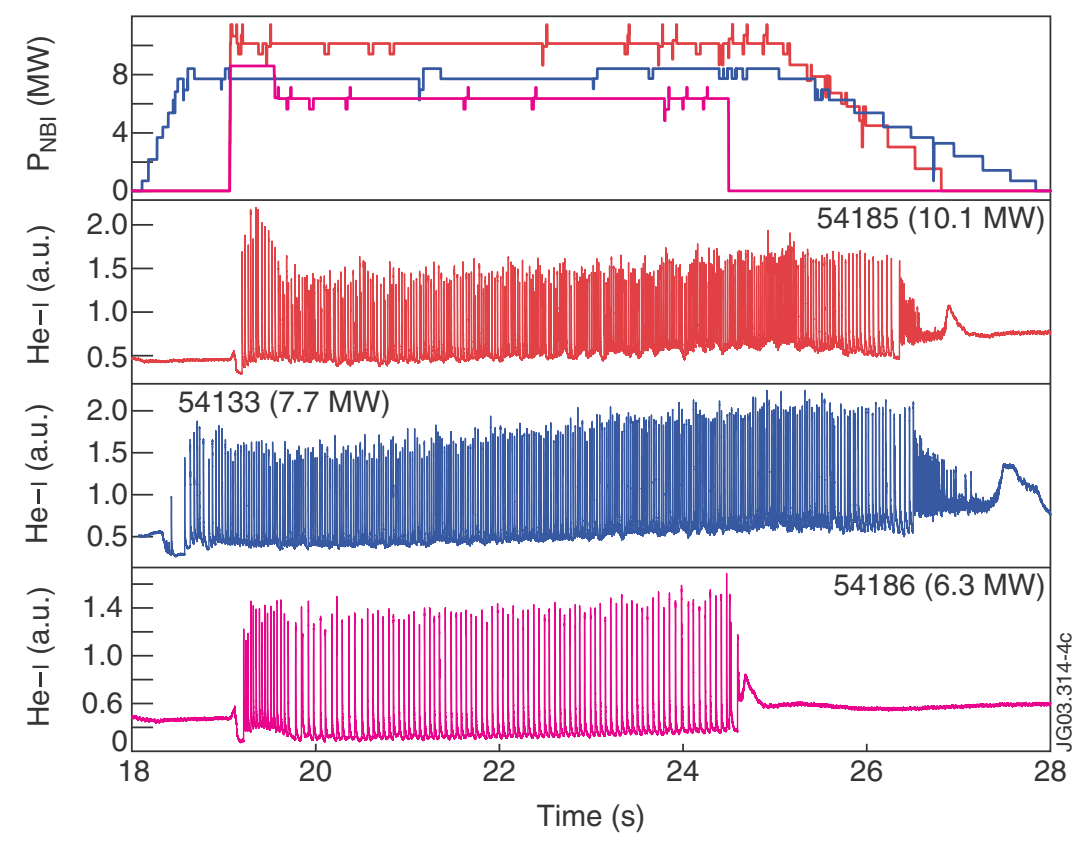

Figure 4. Time traces of three helium-4 ELMy H-mode discharges in a $1 \mathrm{MA} / 1 \mathrm{~T}$ NBI power scan. The signals shown are the injected NBI power (MW) and the He I line emission from the outer divertor (arbitrary units). 


\subsection{Type III ELMS}

At lower powers and/or lower densities, a second ELM regime was observed. The observed ELM frequencies $(30-170 \mathrm{~Hz})$ were higher than for the Type I ELMs, but the He I amplitudes at the crashes were smaller and showed less clearly defined peaks. The energy confinement of the plasmas in these regimes was also lower than that for Type I ELMs (see section 5). In several helium-4 discharges, with decreasing power or increasing density, a direct transition from the Type I to the second regime was observed, with an abrupt change in ELM frequency and amplitude. As all these features are common to Type III ELMs observed in hydrogenic JET plasmas [29,30], they shall be identified here as Type III ELMs in helium-4.

However, the response of the frequency of Type III ELMs in helium-4 to power, often taken as the signature of Type III ELMs in hydrogenic plasmas, is different. Figure 5 shows the results of a NBI only power scan experiment for a $1.5 \mathrm{MA} / 1.5 \mathrm{~T}$ helium-4 plasma in a low triangularity $(\delta \approx 0.2$ ), high septum clearance (X-point $20 \mathrm{~cm}$ above the septum) configuration. Once again, as the NBI power is raised (from 6.0 to $9.7 \mathrm{MW}$ ), the ELM frequency rises monotonically (from 80 to $160 \mathrm{~Hz}$ ). This is the reverse of the trend observed in Type III ELMs in hydrogenic plasmas $[29,30]$ and was observed in all helium-4 Type III ELMy H-mode discharges where the power was varied (six discharges). Although, no specific experiment to study the Type III to ELM free transition was performed, two discharges did show this transition. Although, in both cases the injected power was switched on directly, without a ramp, it is reasonable to assume that the power through the separatrix increased on the timescale of the beam slowing down time and the energy confinement time. In both discharges, then, the ELM frequency (in the range 1-2 kHz) increased with input power, consistent with the above trend for Type III ELMs in helium-4, and then fell discontinuously before the ELM free period.
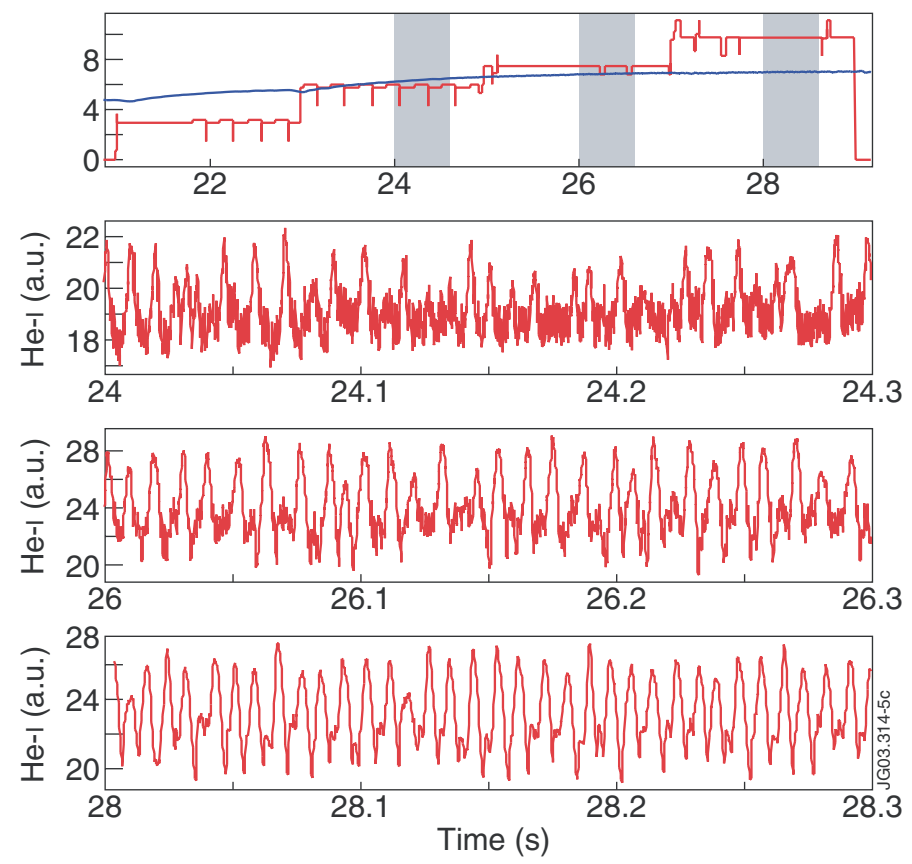

Figure 5. Time trace of JET pulse \#54144, a 1.5 MA/1.5 T NBI heated ELMy H-mode helium-4 plasma. The signals shown are the injected NBI power (NBI) and the He I line emission from the outer divertor (arbitrary units). 


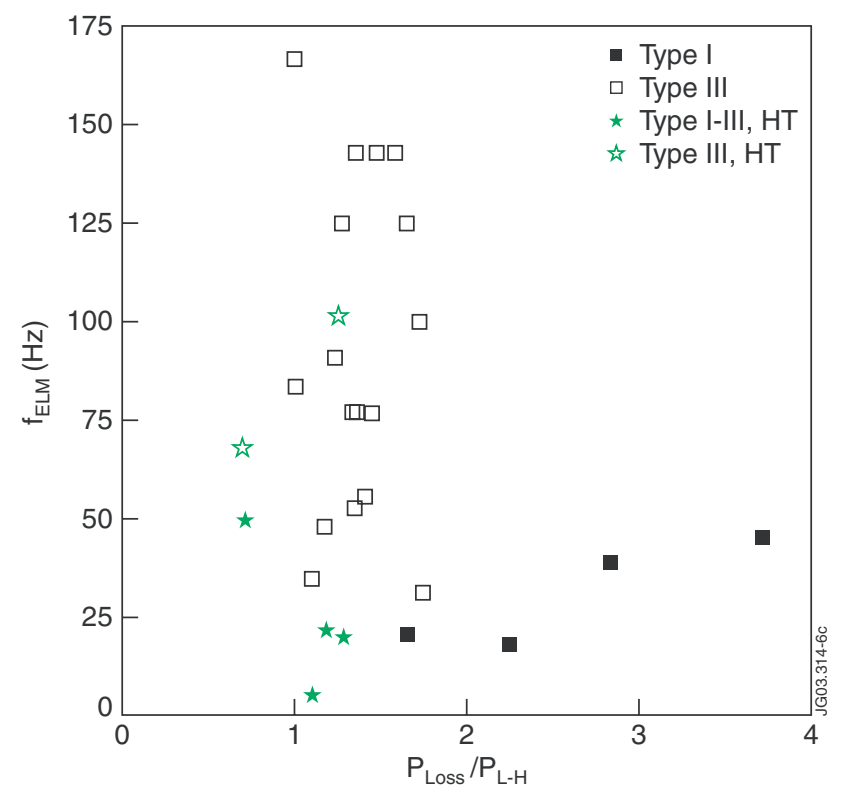

Figure 6. ELM frequency versus loss power through the separatrix, scaled to the helium L-H threshold power (3), for the full database of helium-4 ELMy H-modes. Green stars denote the high triangularity $(\delta \approx 0.4)$ configurations and black squares the more standard triangularity ( $\delta=0.2-0.3)$ discharges. Open symbols denote Type III ELMy H-modes in helium-4, the solid black squares the Type I ELMy H-modes in low triangularity discharges, and the solid green stars the compound Type I-III ELMy H-modes in high triangularity discharges.

Figure 6 shows the relation between the ELM frequency and the loss power relative to the predicted loss power for the L-H transition in helium-4 (equation (3)), for a database of helium-4 Type I, Type III and Type I-III discharges. As for JET deuterium plasmas [32], all the Type III ELMy H-mode discharges have loss powers below twice that of the $\mathrm{L}-\mathrm{H}$ threshold. Similarly, Type I ELMy H-modes are seen at higher powers $\left(P_{\text {loss }}>1.6 P_{\mathrm{L}-\mathrm{H}}\right.$ in all cases). The wide scatter in ELM frequency for the Type III ELMy H-modes implies that parameters other than power, such as plasma current, field, density and configuration, affect the ELM frequency. For the high triangularity $(\delta=0.4)$ plasmas, Type III ELMs were seen at generally lower powers $\left(P_{\text {loss }} / P_{\mathrm{L}-\mathrm{H}}=0.7-1.2\right)$, and a set of compound Type I-III ELMy $\mathrm{H}$-mode plasmas were observed at loss power levels between $10 \%$ and $30 \%$ above the $\mathrm{L}-\mathrm{H}$ threshold, suggesting a somewhat lower Type I-III transition threshold. This is consistent with the effect of triangularity observed in JET deuterium discharges [33, 32].

\subsection{Sawteeth}

Although there is no generally accepted formula or empirical scaling for the sawtooth period of a plasma, de Vries et al [34] found the following fit to the sawtooth period in milliseconds, $\tau_{\text {sawtooth }}^{\text {Scal, }}$, for a range of JET Mark IIGB deuterium plasmas with NBI only auxiliary heating:

$$
\tau_{\text {sawtooth }}^{\text {Scal, }}=20 n_{\mathrm{e} 0}^{0.23} T_{\mathrm{e} 0}^{1.69}
$$

where $n_{\mathrm{e} 0}$ is the core electron density in $10^{19} \mathrm{~m}^{-3}$ and $T_{\mathrm{e} 0}$ the core electron temperature in kiloelectronvolts, are measured immediately before the sawtooth crash. Both the Type I helium-4 ELMy H-modes and their deuterium references give a reasonable fit to this scaling 


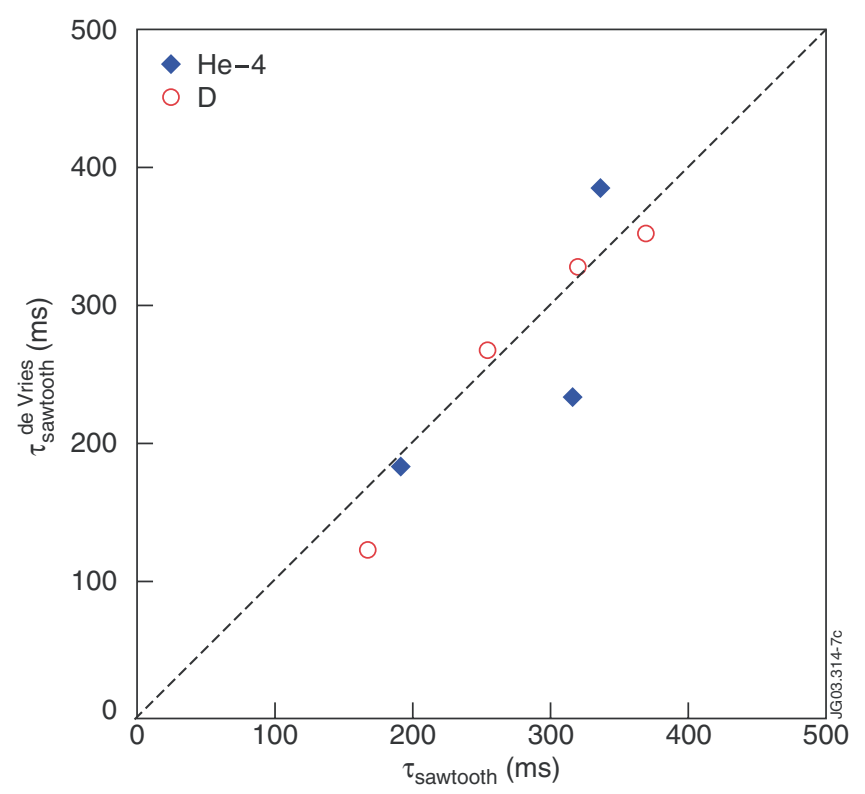

Figure 7. Comparison of the sawtooth period with the scaling law of de Vries et al [34] (6) for helium-4 Type I ELMy H-modes (solid blue diamonds) and their deuterium references (open red circles). The density and temperature are both taken from LIDAR Thomsen scattering, resulting in a time averaged signal, which is multiplied by $10 \%$ to approximate the peak measurements.

(figure 7) with very little sign of an isotope dependence in the absolute value. A direct fit of the data to (5) gives

$$
\frac{\tau_{\text {sawteeth }}^{\text {He-4 }}}{\tau_{\text {sawteeth }}^{\text {Scal, }}}=(0.98 \pm 0.11) \frac{\tau_{\text {sawteeth }}^{\mathrm{D}}}{\tau_{\text {sawteeth }}^{\text {Scal, }}} .
$$

It has also been found that large, ICRH driven, fast energy helium-4 populations can stabilize sawteeth [35], in the same manner as in deuterium. Neoclassical tearing modes, which can be triggered by sawteeth on JET and are known to affect energy confinement [12], were not seen in any of the helium- 4 or deuterium discharges analysed in this paper.

\section{Energy confinement}

The energy confinement of the Type I and Type III ELMy H-modes in helium-4 discharges of section 4 has been studied. As discussed in section 2, the relative inefficiency of the Mark IIGB divertor at pumping helium-4, when compared to deuterium, led to a continual rise in helium- 4 neutral density throughout the discharges. Figure 8 shows a typical helium- 4 Type I ELMy H-mode confinement discharge, with the divertor neutral density increasing throughout the auxiliary heating phase, as seen by the rise in the edge divertor light. This is associated with a rise in line averaged density and a fall in the ELM frequency. An 'edge fuelling steadiness' criterion of $\tau_{\text {divlight }}<5 \tau_{E}$, where $\tau_{\text {divlight }}$ is the time taken for the He I divertor light measurement to double in magnitude, was introduced to ensure that the edge fuelling of the pulses used was steady over several confinement times. $Z$, the mean ion charge was used to describe the relative concentrations of helium- 4 and deuterium in the plasmas. 


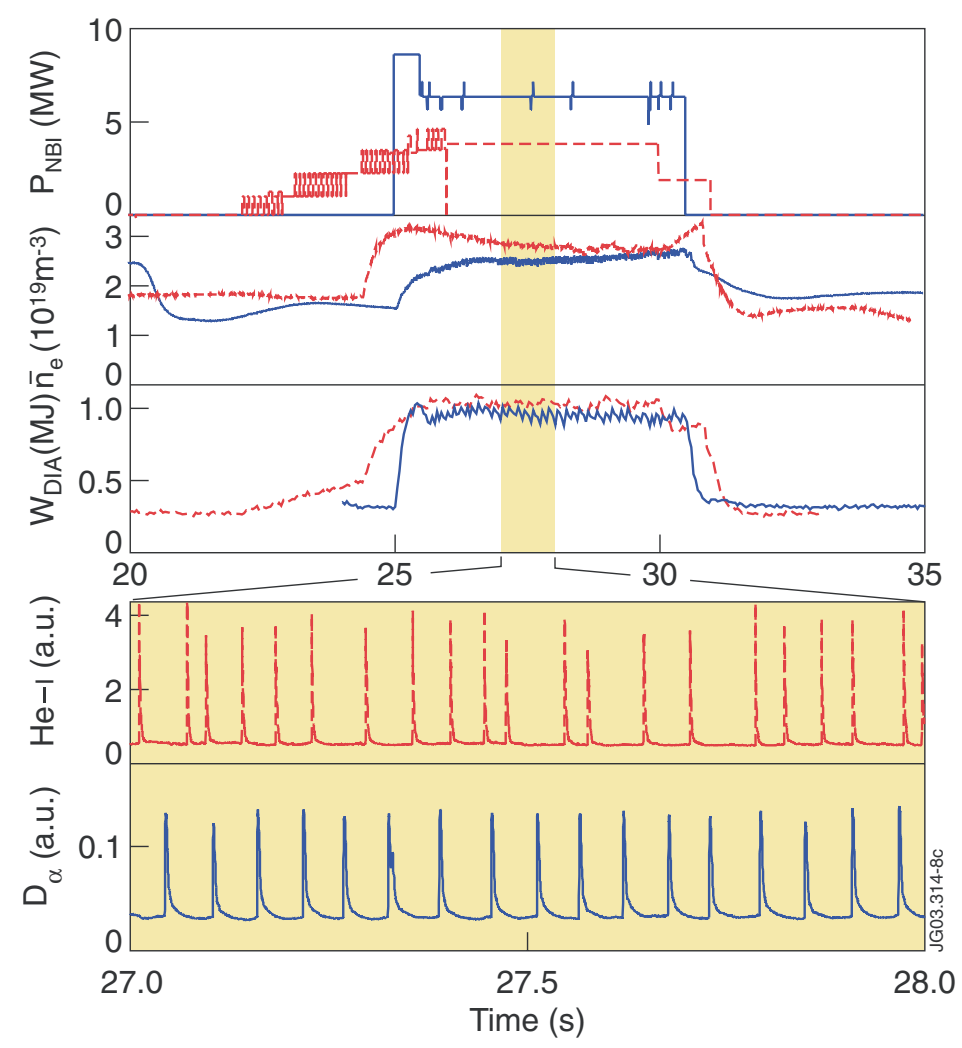

Figure 8. Time trace for a NBI heated Type I ELMy H-mode helium-4 discharge (JET pulse \#54186; solid blue line) and its deuterium reference (JET pulse \#52949; broken red line), both with 1 MA plasma current and $1 \mathrm{~T}$ toroidal field. The signals shown are the injected NBI power (MW), line averaged electron density (calculated from interferometry), stored energy (MJ), and outer divertor light from the $\mathrm{D}_{\alpha}$ line (for deuterium) and the $\mathrm{He}$ I line (for helium-4).

\subsection{Type I ELMs}

Four steady, Type I ELMy H-modes were produced, all at $q_{95} \approx 3.3$, with plasma currents of 1 and $2 \mathrm{MA}$, and densities in the range 55-75\% of the Greenwald density limit [36] (the H-mode density limit in helium-4 ELMy H-modes being close to that seen in deuterium [37]). Helium-4 NBI heating (6-11 MW) was used with some additional second harmonic hydrogen minority ICRH (2 MW) in one discharge. The relatively low number of discharges meant that the dependence of helium-4 energy confinement on current, density and power could not be obtained. However, as a set of reference deuterium plasmas were produced, it is possible to study the isotope dependence.

Figure 8 shows the time evolution of one of the helium-4, 1 MA/1 T, Type I ELMy $\mathrm{H}$-modes compared with that of its deuterium reference. It can be seen that the higher $\mathrm{H}$-mode threshold in helium-4, discussed in section 3, means that a higher input power was required to achieve Type I ELMs for the helium-4 discharge. The resulting plasmas were, however, well matched for electron density and ELM frequency. The energy confinement, for a wide range of hydrogenic plasmas, has been shown to be consistent with the IPB98( $y, 2)$ scaling [12],

$$
\tau_{\text {IPB98 }(y, 2)}=5.62 \times 10^{-2} P_{\text {loss }}^{-0.69} B_{0}^{0.15} I_{\mathrm{p}}^{0.93} \kappa^{0.78} \bar{n}_{\mathrm{e}}^{0.41} a^{0.58} R^{1.39} M^{0.19},
$$




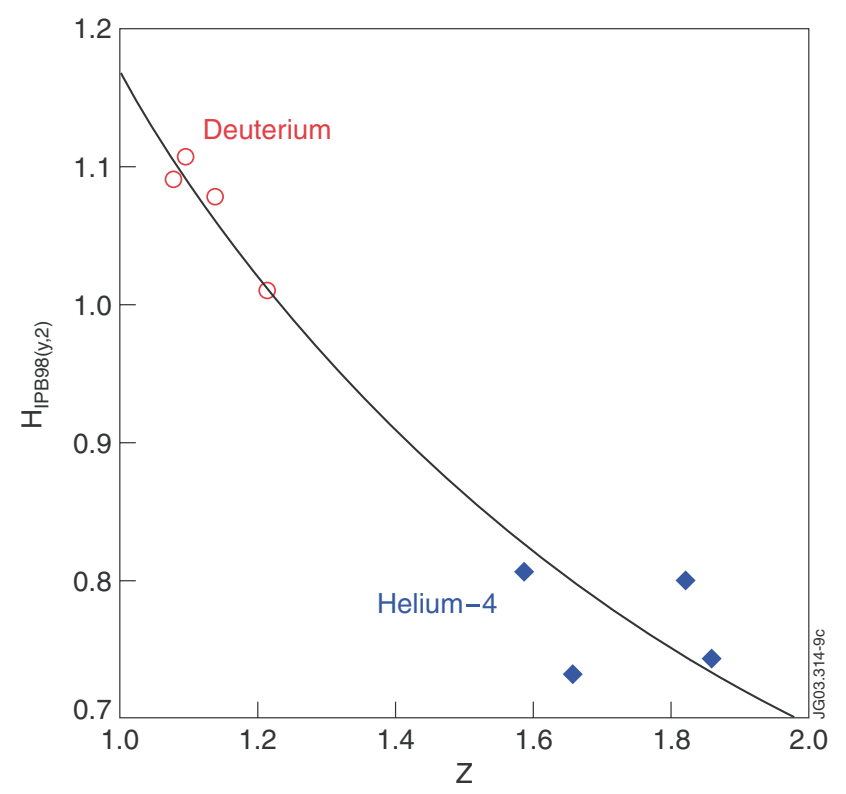

Figure 9. Dependence of the energy confinement time on mean isotope charge for JET Mark IIGB helium-4 (solid blue diamonds) and deuterium reference (open red circles) Type I ELMy H-mode plasmas. The confinement time is normalized to the ELMy H-mode scaling IPB98( $y, 2)(7)$. The solid line represents the fit of equation (8).

where $\tau_{\mathrm{IPB} 98(y, 2)}$ is the energy confinement time in seconds. The mass dependence means that the scaling predicts that energy confinement in helium-4 plasmas would be $14 \%$ higher than in deuterium. It can be seen from figure 8 , however, that this was not the case, with the stored energy for the helium- 4 plasma lying below that of its deuterium reference, despite the $70 \%$ higher injected power.

Figure 9 shows the energy confinement time, scaled to $\operatorname{IPB} 98(y, 2)$, for the full set of helium-4 Type I ELMy H-modes and their deuterium references. All discharges are in a low triangularity $(\delta \approx 0.2)$, standard septum clearance (X-point $8 \mathrm{~cm}$ above the septum) configuration with $q_{95}=3.3$, and $I_{\mathrm{p}}=1-2.6 \mathrm{MA}$. The deuterium references themselves contain concentrations of helium-4 (9-21\%), due to helium-4 left in the machine after helium glow discharge cleaning. The helium- 4 discharges can clearly be seen to have lower energy confinement with a mean scaled energy confinement time $\left(H_{\operatorname{IPB} 98(y, 2)}=\tau_{E} / \tau_{\operatorname{IPB} 98(y, 2)}\right)$ of $0.77 \pm 0.04$ compared with $1.07 \pm 0.04$ for the deuterium discharges. This can be represented by introducing a mean ion charge dependence of the form,

$$
\tau_{E}=Z^{-0.75 \pm 0.08} \tau_{\mathrm{IPB} 98(y, 2)},
$$

where it should be remembered that the $\operatorname{IPB} 98(y, 2)$ scaling has a mass dependence of $M^{0.19 \pm 0.05}$. As with the L-H power threshold, it may be that a power law dependence on isotope charge is inappropriate for describing the energy confinement in plasmas of different isotopes. However, as plasma energy transport is believed to be dominated by the physics of fully ionized plasmas, it would appear to be a reasonable assumption.

The energy confinement time of a pure helium-4 plasma relative to a pure deuterium plasma with all other engineering plasmas, including electron density, constant is thus estimated as $\tau_{E}^{\mathrm{He}-4} / \tau_{E}^{\mathrm{D}}=0.68 \pm 0.08$. Figure 10 shows the confinement time for a set of discharges that include hydrogen and tritium plasmas [38]. The energy confinement time has been normalized 


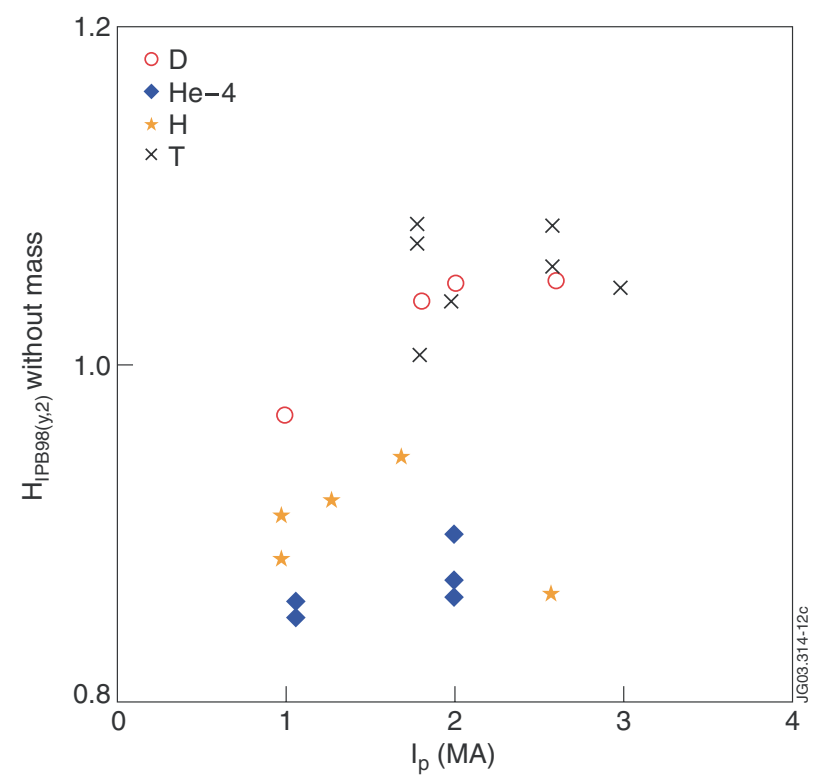

Figure 10. Confinement time, normalized to then ELMy H-mode scaling IPB98( $y, 2)(7)$, divided by $(M / 2)^{0.19}$ to remove the mass dependence, for JET Mark IIa and Mark IIGB hydrogen (solid yellow stars), deuterium (open red circles), tritium (black crosses) and helium-4 (solid blue diamonds) Type I ELMy H-mode plasmas in the same configuration.

to the IPB98 $(y, 2)$ scaling, with the isotope mass dependence removed to enable the absolute plasma performance of each isotope species to be compared. Once again, all discharges are in a low triangularity $\left(\delta \approx 0.2\right.$ ) configuration with $q_{95}=3.3$ and $I_{\mathrm{p}}=1-2.6 \mathrm{MA}$. It should be noted, however, that the hydrogen and tritium data are taken from experiments performed in the JET MkIIa divertor. As can be seen from the figure, energy confinement in helium-4 plasmas lies below that of hydrogen plasmas. Thus, for the low activation phase of a next step machine, hydrogen plasmas would be expected to have better performance.

\subsection{Type III ELMs}

Figure 11 shows $H_{\mathrm{IPB} 98(y, 2)}$ for the full JET database of steady helium-4 ELMy H-modes. The fall in confinement, as one moves from Type I (mean $\left.H_{\mathrm{IPB} 98(y, 2)}=0.77 \pm 0.04\right)$, to compound Type I-III (mean $\left.H_{\mathrm{IPB} 98(y, 2)}=0.54 \pm 0.03\right)$ and Type III $\left(\right.$ mean $\left.H_{\mathrm{IPB} 98(y, 2)}=0.46 \pm 0.05\right)$ ELMy $\mathrm{H}$-modes, is clear. Correcting for the isotope charge scaling, using equation (8), helium-4 Type III ELMy H-modes have an energy confinement time that is $35 \%$ of that seen for helium-4 Type I ELMy H-modes. This is a somewhat larger fall than is usually observed in deuterium H-modes at JET [32].

\subsection{Non-dimensional parameters}

The difference in confinement between hydrogenic and helium-4 plasmas can also be expressed in terms of non-dimensional parameters. For a given configuration and $q$-profile, single ion species equipartioned plasmas dominated by high- $\beta$ transport have been shown theoretically [39-41] to have a normalized confinement time, $\omega_{\mathrm{ci}} \tau_{E}$, where $\omega_{\mathrm{ci}}$ is the ion Larmor frequency, which is a function of the non-dimensional plasma parameters $\rho_{\mathrm{i}}^{*}, \beta$ and 


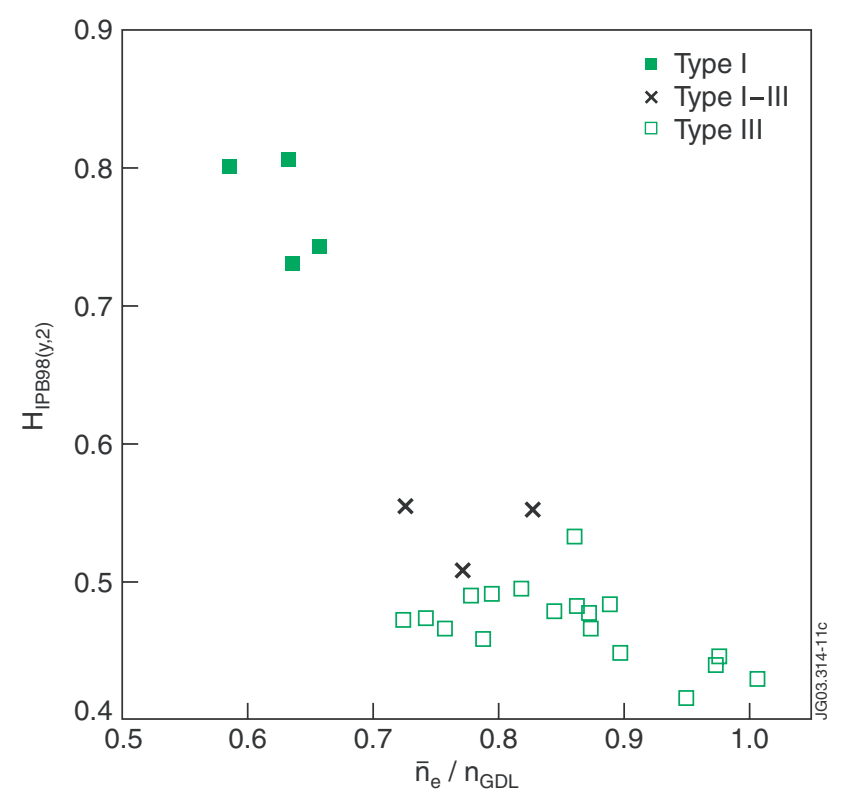

Figure 11. Energy confinement time, normalized to the ELMy H-mode scaling IPB98( $y$, 2) (7), against line averaged density scaled to the Greenwald density limit. Closed green squares denote Type I ELMy H-modes, black crosses denote compound Type I-III ELMy H-modes, and open green circles denote Type III ELMy H-modes in helium-4.

$v_{\mathrm{ei}}^{*}$ (see Connor and Taylor [41] for definitions). If the effects of the isotope on the system are included, it is found that no new invariant transforms appear. This may be thought of as being due to the independent $Z$ and $M$ scaling in the ion-ion, electron-electron and ion-electron collisionality. Thus, the energy confinement may be expressed as

$$
\omega_{\mathrm{ci}} \tau_{E}=F\left(\rho_{\mathrm{i}}^{*}, \beta, v_{\mathrm{ei}}^{*}, Z, M ; \varepsilon, q, \kappa, \delta, \frac{T_{\mathrm{i}}}{T_{\mathrm{e}}}, \ldots\right),
$$

where, although the ion Larmor frequency, Larmor radius and ion-electron collision time have been chosen, taking their electron equivalents would merely change the functional dependence on $Z$ and $M$. This means that identity experiments between different isotopes cannot be performed even with matched $\rho_{\mathrm{i}}^{*}, \beta$ and $\nu_{\mathrm{ei}}^{*}$. However, the functional dependence of equation (9) in terms of $Z$ and $M$, can be determined.

Log-linear fits to a multi-machine database [12] of hydrogenic ELMy H-modes, have shown that the mass dependence may be represented by the scaling

$$
\omega_{\mathrm{ci}} \tau_{E}=\rho_{\mathrm{i}}^{-2.70} \beta^{-0.90} v_{\mathrm{ei}}^{-0.01} M^{0.96} q^{-3.0} \varepsilon^{0.73} \kappa_{a}^{2.3} G\left(Z ; \delta, \frac{T_{\mathrm{i}}}{T_{\mathrm{e}}}, \ldots\right),
$$

where, $\kappa_{a}=S / \pi a^{2}$ is the elongation and $S$ is the area of the poloidal cross section. However, it should be noted that JET experiments with hydrogen and deuterium, which matched $\rho_{\mathrm{i}}^{*}$, $\beta$ and $\nu_{\mathrm{ei}}^{*}$ [40], suggested a much weaker isotope mass dependence. Assuming the form of equation (10), and looking for a power law form for the isotope charge dependence, the best fit to the helium-4 Type I ELMy H-modes and their deuterium references gives

$$
G\left(Z ; \delta, \frac{T_{\mathrm{i}}}{T_{\mathrm{e}}}, \ldots\right)=Z^{-1.54 \pm 0.38} H\left(\delta, \frac{T_{\mathrm{i}}}{T_{\mathrm{e}}}, \ldots\right),
$$

where it should be noted that the dilution of the hydrogenic and helium- 4 plasmas has been included in the calculation of $\beta$ and $\nu_{\mathrm{ei}}^{*}$. 


\section{Summary}

A series of single null, diverted helium-4 H-mode plasmas were performed at JET to improve the understanding of $\mathrm{H}$-mode physics and to investigate their suitability for low activation phases in future tokamak designs, such as ITER.

Argon frosting on the Mark IIGB divertor enabled helium-4 to be pumped successfully. NBI, with sources converted to helium- 4 and argon frosting of the cryopanels between discharges, provided up to $12 \mathrm{MW}$ of auxiliary heating. Deuterium removal from the near surface wall reservoir [5] meant that helium-4 L-H threshold and Type III ELMy H-mode experiments had high purity (84-95\%), although Type I ELMy H-mode purity was lower $(65-86 \%)$.

The L-H power threshold in helium- 4 has been shown to have a similar dependence on density and field as for deuterium plasmas, but with an absolute value $42 \pm 10 \%$ higher than for a set of deuterium references. This, together with earlier experiments with hydrogenic species [23], is consistent with a $\mathrm{P}_{\mathrm{L}-\mathrm{H}} \propto M^{-1.1} Z^{1.6}$ isotope scaling. Kiveniemi et al [25] and Hidalgo et al [26] have shown this to be in qualitative agreement with models based on shear flow stabilization of edge turbulence. The $\mathrm{L}-\mathrm{H}$ threshold in helium-4 was also shown to fall, by up to $50 \%$, as the $\mathrm{X}$-point is lowered on to the septum. This is consistent with observations in deuterium [27] and suggests that neutral penetration is important in $\mathrm{L}-\mathrm{H}$ transition physics.

Both the Type I ELMy H-mode regime and a second ELMy H-mode regime, identified here as the Type III ELMy H-mode in helium-4, have been explored which, for the most part, show the same ELM crash characteristics and occupy the same region of loss power space relative to the $\mathrm{L}-\mathrm{H}$ threshold. The exception is that the ELM frequency increases with increasing power for Type III ELMy H-modes in helium-4, the reverse of the deuterium trend. Energy confinement in helium-4 has $H_{\mathrm{IPB} 98(y, 2)}=0.77 \pm 0.04$ for Type I ELMy H-modes, compared with $H_{\operatorname{IPB} 98(y, 2)}=1.07 \pm 0.04$ for the deuterium reference discharges. This has been expressed by the addition of an isotope charge dependence to the ELMy H-mode energy confinement scaling, of the form $\tau_{E}=Z^{-0.75 \pm 0.08} \tau_{\mathrm{IPB} 98(y, 2)}$. In dimensionless coordinates, $\tau_{E}$, normalized by $\omega_{\mathrm{ci}}$, is proportional to $M^{0.96} Z^{-1.54}$ for fixed $\rho_{\mathrm{i}}^{*}, \beta$ and $\nu_{\mathrm{ei}}^{*}$. For Type III ELMy H-modes in helium-4 plasmas $H_{\mathrm{IPB} 98(y, 2)}=0.46 \pm 0.05$.

As far as early operation of ITER in helium-4 is concerned JET has demonstrated that Type I and Type III ELMy H-modes can be sustained for many confinement times in helium-4. The $\mathrm{L}-\mathrm{H}$ power threshold in helium-4 (1.42 times that of deuterium) has been shown to be lower than that in hydrogen (2.0 times that of deuterium), the alternative isotope. This would enable an increased range of field and density to be explored. However, the lower energy confinement in helium-4 (0.68 times that of deuterium) compared with hydrogen ( 0.87 times that of deuterium) would result in lower normalized pressures.

\section{Acknowledgments}

This work has been conducted under the European Fusion Development Agreement and is partly funded by EURATOM and the United Kingdom Engineering and Physical Sciences Research Council.

\section{References}

[1] West W P et al 1999 J. Nucl. Mater. 266-269 732

[2] Pitts R A et al 2003 J. Nucl. Mater. 313-316 777 
[3] Huber A et al 2003 Tomographic reconstruction of 2-D line radiation distribution in the JET MkIIGB divertor in pure He and D discharges Proc. 30th EPS Conf. on Controlled Fusion and Plasma Physics (St Petersburg, 2003) ECA vol 27A P-1.162

[4] Mantsinen M J et al 2001 ICRF heating scenarios in JET with emphasis on ${ }^{4} \mathrm{He}$ plasmas for the non-activated phase of ITER Radio-Frequency Power in Plasmas: Proc. 14th Topical Conf. on Radio Frequency Power in Plasmas (Oxnard, CA, USA, 7-9 May 2001) (New York: AIP) pp 59-66

[5] Hillis D L et al 2003 J. Nucl. Mater. 313-316 1061

[6] Fenstermacher M E et al 2003 J. Nucl. Mater. 313-316 1206

[7] Challis C et al 1989 Nucl. Fusion 29563

[8] O'Mullane M et al 2003 Advanced Diagnostics for Magnetic and Inertial Fusion ed P E Stott et al (New York: Plenum) p 67

[9] Zastrow K-D, Brix M, Giroud C, Meigs A, O’Mullane M G, Proschek M and Summers H P 2003 Plasma Phys. Control. Fusion 451747

[10] Morgan P D and Contributors to the EFDA-JET Workprogramme 2003 Advanced Diagnostics for Magnetic and Inertial Fusion ed P E Stott et al (New York: Plenum) p 403

[11] Suttrop W et al 2002 Testing H-mode parameter similarity in JET and ASDEX Upgrade Proc. 29th EPS Conf. on Controlled Fusion and Plasma Physics (Montreux, 2002) ECA vol 26B P-1.030

[12] ITER Physics Expert Group 1999 Nucl. Fusion 392175

[13] Ryter F and the H-Mode Database Working Group 1996 Nucl. Fusion 361217

[14] Snipes J A and the International H-mode Threshold Database Working Group 2000 Plasma Phys. Control. Fusion 42 A299

[15] Ryter F and the H-mode Threshold Database Group 2002 Plasma Phys. Control. Fusion 44 A415

[16] Mori M et al 1994 Plasma Phys. Control. Fusion 36 A39

[17] Ryter F et al 1994 Plasma Phys. Control. Fusion 36 A99

[18] Fielding S J et al 1996 Plasma Phys. Control. Fusion 381091

[19] Snipes J A et al 1996 Plasma Phys. Control. Fusion 381127

[20] Calstrom T N and Groebner R J 1996 Phys. Plasmas 31867

[21] Righi E et al 2000 Plasma Phys. Control. Fusion 42 A199

[22] Horton L D et al 1999 Dependence of the H-mode Threshold on the JET Divertor Geometry (Maastricht, 1999) vol 23J (ECA) p 193

[23] Righi E et al 1998 Plasma Phys. Control. Fusion 40721

[24] Righi E et al 1999 Nucl. Fusion 39309

[25] Kiviniemi T P et al 2003 L-H transition threshold temperature for helium discharges in JET 30th EPS Conf. on Controlled Fusion and Plasma Physics (St Petersburg, 2003) ECA vol 27A P-1.162

[26] Hidalgo C et al 2003 J. Nucl. Mater. 313-316 863

[27] Righi E 2000 Transition to H-mode regime in JET with and without pumped divertors Proc. 18th IAEA (4-10 October 2000) paper IAEA-CN-77/EXP5/31

[28] Horton L D 2000 Plasma Phys. Control. Fusion 42 A37

[29] Doyle E J et al 1991 Phys. Fluids B 32300

[30] Zohm H 1996 Plasma Phys. Control. Fusion 38105

[31] Loarte A et al 2003 Plasma Phys. Control. Fusion 451549

[32] Sartori R et al Study of Type III ELMs in JET Plasma Phys. Control. Fusion Submitted

[33] Sartori R et al 1999 Confinement loss in JET ELMy H-modes (Maastricht, 1999) vol 23J (ECA) p 197

[34] de Vries P C, Pochelon A, Johnson M F, Nave M F F, Howell D F, Sauter O and Contributors to the EFDA-JET Workprogramme 2001 Analysis of shaping effects on sawteeth in JET Proc. 28th EPS Conf. on Controlled Fusion and Plasma Physics (Madeira, 2001)

[35] Mantsinen M J et al 2002 Phys. Rev. Lett. 88105002

[36] Greenwald M et al 1988 Nucl. Fusion 282199

[37] Rapp J et al 2003 J. Nucl. Mater. 313-316 524

[38] Saibene G et al 1999 Nucl. Fusion 391133

[39] Kadomstev B B 1975 Sov. J. Plasma Phys. 1295

[40] Cordey J G et al 1996 Plasma Phys. Control. Fusion 38 A67

[41] Connor J W and Taylor J B 1977 Nucl. Fusion 171047 